\title{
TDR Liquid Level Detection Program
}

by

G. Reeves

Westinghouse Savannah River Company

Savannah River Site

Aiken, South Carolina 29808

B. Toole

Point of Contact, 952-1864

DOE Contract No. DE-AC09-89SR18035

This paper was prepared in connection with work done under the above contract number with the U.S.

Department of Energy. By acceptance of this paper, the publisher and/or recipient acknowledges the U.S.

Government's right to retain a nonexclusive, royalty-free license in and to any copyright covering this paper, along with the right to reproduce and to authorize others to reproduce all or part of the copyrighted paper. 


\section{DISCLAIMER}

Portions of this document may be illegible in electronic image products. Images are produced from the best available original document. 
ABSTRACT

1a. Software Acronym:

1b. Short Title: $\quad$ TDR Liquid Level Detection Program

2.Author Name(s) and Affiliations:

Greg Reeves, WSRC

. '

\section{Software Completion Date: $\quad 8 / 1 / 90$}

4. Brief Description:

A data acquisition system for monitoring the SRS reactor Supplemental Safety Systme (SSS) during chargeback testing has been installed. The system is based on liquid level detection by means of time domain reflectometry. Software developed for the system allows data to be collected from a digitizing oscilloscope by an IBM AT computer, displayed and stored as needed by the user. The software is menu-driven to facilitate user interaction.

5.Method of Solution:

Converts signals from a Hewlett Packard oscilloscope with a TDR head. The TDR device works by sending a sharp electric pulse down a probe to the bottom of a tank. As pulse reaches different interfaces along the probe part of it is reflected back.

6.Computer(s) for which software is written:

IBM At computer/compatibles

7. Operating System:

MS DOS 


\section{ABSTRACT}

8. Programming Lanaguage(s) Used:

Microsoft C

9. Software Limitations:

Must have a CEC 488 card in stalled.

10. Unique,features of the Software:

Using an IBM computer to control and evaluate TDR technology to determine and monitor liquid level makes this software novel and unique.

11. Related and Auxiliary Software:

none

12. Other Programming or Operating Information or Restrictions:

CEC library

13. Hardware Requirements:

CEC 488 Card

14. Time Requirments:

none

15. References:

TDR Liquid Level Detection Program Software Usre Manual

16a. Subject Classification Code:

16b. Keywords: 
TDR LIQUID IEVEL DETECTION PROGRAM

SOFTTARE USER MANUAL

TABIE OF CONTENTS

1. START-UP

2. STARTING FROM DOS

3. MENU SELECTION

4. PRETEST SAMPLING.

5. SAMPLING

6. MAIN MENU

6.1. RUN

6.2. CHANGE BASE LEVEL

6.3. GRAPH

6.4. SAVE DATA TO FEOPPY DISKETTE

1

2

2

3

5

6

6

6

7 


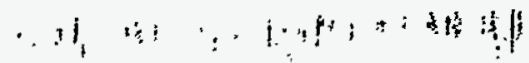

TDR LIQUID LEVEL DETECTION PROGRAM Software User Manual

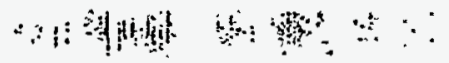

page 1 of 9 :

July 1, 1996

TDR LIOUID LEVEL DETECTION PROGRAM

BOFTWARE USER MANUAL

4

The software developed for the Time Domain Reflectometry (TDR) Liquid Level Detector provides a menu-driven data acquisition system. The system collects and displays liquid level data in inches, plots the data, and stores the data on magnetic media. The data is acquired from a Hewlett Packard 54120B Digitizing oscilloscope by an IBM computer.

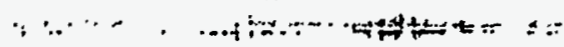

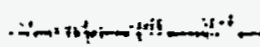

\section{START-UP}

The system is setup to automatically change to the directory containing the 'TDR IIQUID LEVEL DETECTION 'program 'when the computer comes on. Be sure that there is not a diskette in disk drive $A$ : (the drive on the right side) or the system will boot from the $A$ : drive instead of the hard disk. The computer and other devices should be turned on as follows:

1) Turn on the HP 54120B Digitizing Oscilloscope.

2). Be sure that the oscilloscope's time delay is set so that the waveform representing the TDR probe connector is near the left side of the screen and the screen offsets and division sizes are set so that the whole waveform for the probe will show on the scope screen. The scope TIMEBASE and CHANNEL menus (selected using the keys below the scope:screen and then the keys to the right of the scope screen) are used to adjust the waveform on the screen, Refer to the Hewlett Packard 54120B Digitizing Oscilloscope manuals for directions in using the scope. The scope will require time to warm-up and will work best if turned on at least a half hour. before a test.

3). Turn on the computer. Start the TDR program as described in the STARTING FROM DOS section of this manual.

4). Check the version number displayed on the screen to ensure that the correct version of the software is being used. Press any key to begin the Pre-test sampling. 
5). Check the values shown on the computers screen and verify that the level reading shown is reasonable. Make sure that the voltage markers (horizontal dashed lines) on the oscilloscope are positioned so that the connector and liquid level will be detected properly.

6). Leave the program-on the PRETEST SAMPIING screen (the screen that is displayed after the title screen).

\section{STARTING FROM DOS}

The program can be started from the disk operating system when a DOS prompt is showing. The active directory must be the TDR .program directory _...To change, to. ,this directory..type:

CD ITDR

and press the ENTER key. ." $\therefore$. . .

To start the program, type the command:

TDR

and press the ENTER key. The TDR program will be loaded from the hard drive.

The keyboard is locked if the system unit's disk drive door is locked. If the computer does not respond to keys pressed, check and be sure the disk drive door is unlocked and the keyboard is plugged into the system unit. The PRETEST SAMPLING screen will be displayed as described in the PRETEST SAMPIING section.

\section{MENO SELECTION}

The menus use highlighting "and arrow keys to" select menu options. Selections are made by moving the highlight to the choice and then pressing the ENTER key (key in the lower right corner of the keyboard or key above the shift key on the right side of the letters).

The bottom Iine of the screen displays the instructions "ARROWS to select, ENTER to CHOOSE". The menu options are displayed on the lines above the instructions. One of the options will be highlighted (have bright white characters). The highlighted option will be chosen if the ENTER key on the keyboard is pressed. A default option will be highlighted when the menu first appears. To select this default option, press only the ENTER key. To select another option, use either the arrow keys or press the key on the keyboard that 
corresponds to the first letter of the choice until the choice is highlighted.

The RIGHT ARROW key moves the highlight to the next option to the right of the highlighted option. However, if the last option was highlighted, then the first option becomes highlighted.

The LEFT ARROW key moves the highlight to the next option to the left. However, if the first option was highlighted, then the last option becomes highlighted.

The DOWN ARROW key moves the highlight to the last option. The UP ARROW. key moves the highlight to the first option..

Pressing a key on the keyboard that corresponds to the first letter of one of the options will move the highlight to the next option that begins with that letter. 'Pressing a key that does not correspond to the first letter of any option will do nothing.

\section{PRETEST SAMPIING}

When the program is first started and when "RUN" is selected from the main menu, the PRETEST SAMPIING screen will be... displayed as follows:

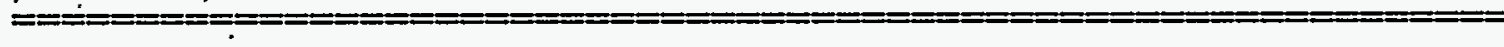

Version: 1.00 Revision date: 6-10-1991

Test will run for 180 seconds after trigger.

Connector voltage Level $=0.22$ volts

Iiquid Level voltage Level $=0.20$ volts

ns per inch $\quad=0.17218$

base level: $\quad 82.0$ inches

PRETEST SAMPLING....

75.3 inches

$<$ ALT> Q -Menu
} 
The screen shown is an example. The values shown will be different for the actual test setup. The top line of this screen shows the version number and the last revision date of the software. The second line shows the length of time (in seconds) that the test will run after a trigger pulse is received by the computer. See the SAMPLING section for more details. The third line of the PRETEST SAMPIING screen shows the connector voltage level. The time at which the waveform first crosses this voltage level in the negative (downward) direction corresponds to the position of the connector. The fourth line shows the liquid level voltage. The time at which the waveform first crosses this voltage level in the negative (downward) direction corresponds to the surface of the liquid. The fifth line shows the nanoseconds per inch used to convert the..time. read from the scope to the level in inches. The next line shows the base level. The base level is the distance in inches from where the system detects the connector to where the system detects the bottom of the probe. The line displaying "PRETEST SAMPLING" indicates that the test has not begun yet. The next line shows the tank level in inches that the computer is currently calculating. The tank level will be continually updated as fast as readings are received from the oscilloscope. The last line indicates the keys to use to bring up the main menu.

-Hold down the alternate key and press the $Q$ key to bring up the main menu.

When <ALT> $Q$ is pressed, before the main menu comes up, the SAVE PRETEST SAMPLING menu may appear as follows:

\section{PRETEST SAMPLES TAKEN:}

No TEST SAMPIES taken.

Save PRETEST sampling data Exit without saving ARROWS to Select, ENTER to CHOOSE

Note that no test data was taken. If the pretest samples are to be saved to the hard disk, then choose the "Save PRETEST sampling data" option. The data will be saved on the hard disk as described for the sample data in the SAMPLING section of this manual. 
-Choose "Exit without saving" and the pretest sampling data will be lost. This is usually the option selected from this screen since the pretest data is not normally of any value.

-Choose "Save PRETEST sampling data" to save the data to the hard disk. The file name that the data is being saved to will be displayed while the data being written to the hard disk....... is

After either option is selected the main menu will be displayed.

Be sure the PRETEST SAMPLING screen is displaÿed when the trigger is expected.

\section{SAMPLING}

When the computer is operating on the PRETEST SAMPLING screen and a trigger pulse is received, the TEST SAMPLING screen wiII be displayed as follows:

Version: 1.00

Revision date: 6-10-1991

Test will run for 180 seconds after trigger.

Connector voltage Level $=0.22$ volts

Liquid Level voltage Level $=0.20$ volts

ns per inch $\quad=0.17218$

base level: $\quad 82.0$ inches

SAMPLING....

75.3 inches

The SAMPLING screen shows the same information as the PRETEST sampling screen. However, when this screen is displayed the test is in progress. The line that had displayed "PRETEST SAMPLING" now displays the message "SAMPLING" to indicate that test data is being taken. Sampling will continue for the length of time indicated on the first line (180 seconds in this example). When sampling is completed, the data will be written to a data file and then graphed on the computer screen. The name of the data file will be composed of the date and time as described in the GRAPH section of this manual. The data file will have a ".TDR" extension to the file name. 
-Do not touch any keys on the computer or oscilloscope when this screen is displayed. There is no key stroke that will stop the computer from taking data. Sampling will stop only when the time has expired.

\section{MAIN MENU}

is

The main menu will display the options "Run", "Change base level", "Graph last data", "Save data to floppy", and "Exit" on the bottom lines of the screen.

\subsection{RUN}

-Choose..!Run"! from the main menu to display the...PRETEST

SAMPLING screen and have the computer watching for the trigger pulse. The "Run" option should always be chosen instead of leaving the computer on the main menu.

\subsection{CHANGE BASE LEVEL}

When "Change base level" is chosen from the main menu, the " CHANGE VOLTAGE LEVEL screen will be displayed as follows:

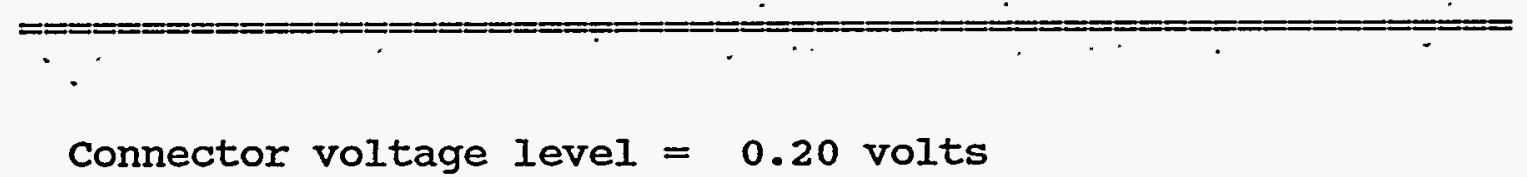

Move Scope Delta V START, MARKER to new level.

START MARKER at new level Exit ARROWS to select, ENTER to CHOOSE

The top line shows the currently set connector voltage level. This is the value that will be changed by this option. The new connector voltage level will be set by reading the position of the V MARKER 1 from the oscilloscope. If the connector voltage level is changed, the liquid level voltage will also be changed to maintain the same difference between the two voltage levels.

-Choose "Exit" to return to the main menu without changing the voltage levels. 
-To change the voltage level:

1). Move the $V$ MARKER 1 on the oscilloscope to the desired voltage level by using either the wheel on the right side of the scope or by typing in the actual voltage on the scopes numeric keypad and then pressing the "volts" key on the scope. The position of $V$ MARKER 1 is shown on the scope as "V(I) $=200 \mathrm{mV}$ in the lower left corner of the screen when on the DELTA $V$ menu. When $V$ MARKER $I$ is the active marker, the position of $V$ MARKER 1 will be shown at the top of the oscilloscope screen. Note that the voltage is given in Millivolts on the oscilloscope.

2) - Choose "START MARKER at new base level" from the CHANGE VOIIAGE IFVEL screen."-Be-sure-that V-MARKER-1-is-at-thedesired position before pressing the ENTER key to choose this option.

3). Verify the new base level by choosing "Set connector voltage level $=0.20$ volts" from the menu that appears. The new level read from the oscilloscope is displayed as part of the menu.

-Choose "Abort" to return to the main menu without changing the base level.

6.3. GRAPH

A graph of the data from the last test performed will be displayed when "Graph last data" is chosen from the main menu and also immediately after completion of a test run.

The graph shows the data just before the trigger was received to begin the test with negative seconds on the time scale ( $X$ axis). The test data begins at time 0 seconds and continues to the end of the graph. The $Y$ axis shows the liquid level. in inches. The title of the graph includes the date and time of the trigger.

The bottom Iines of the graph show the menu options "Select other data" and "Exit".

-Choose. "Exit" and the main menu is displayed. 
If "Select other data" is chosen, the SELECT DATA FILE screen will be displayed as follows:

SELECT DATA FILE

$05220814 . T D R$

05211545.TDR

$05211222 . T D R$

Exit

ARROWS to Select, ENTER to CHOOSE

other data files are displayed as the options. The file names are based on the date and time the test was triggered. For example; the first option shown here ("05220814.TDR") is the data for the test that was triggered on May (05 represents the month) 22 at 8:14. The last option is "Exit". If there are" more files than will fit on the screen at once, the options "NEXT FILES" and "BEGINNING OF DIRECTORY" might also be displayed.

-Choose "Exit" to end the graphing routine and display the main menu.

-Choose the file name and the graph of the data will be displayed with the options at the bottom of the screen as described previously.

-Choose "NEXT FILES" to display more files.

-To start the listing of files over with the first files shown, choose "BEGINNING OF DIRECTORY". 
FILE: tdr.c

07/01/96 14:16:04

$\begin{array}{lc}\text { copy_data } & 4 \\ \text { tdr_test } & 5 \\ \text { create_title } & 11 \\ \text { check_key } & 12 \\ \text { set_base_seconds } & 13 \\ \text { set_pre_screen } & 15 \\ \text { get_flname } & 16 \\ \text { transmit_488 } & 17 \\ \text { send_488 } & 18\end{array}$


\#include 〈stdio.h>

\#include <stdlib.h>

\#include <conio.h>

\#include <dos.h>

\#include 〈bios.h>

\#include <math.h>

Hinclude <graph.h>

\#include "IEEE-C. H"

\#include "menu.h"

\#define NAHO_PER_SEC 1E+9

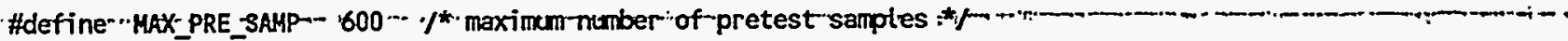

int hp54121t $=7 ; \quad / *$ GPIB bus address of oscilloscope */

struct dostime_t beg_time;

struct dostime_t end_time;

struct dosdate_t date;

char strg[132];

"float time period; $/$ * length of test in seconds *I

,float volt_lev; $\quad l^{*}$ voltage level to read time crossing *f

float base_seconds; $I$ * base level in seconds from delay *J

float base_inches; $I^{*}$ base level in tank inches *

float ns_per_inch; $/ *$ nanoseconds on $x$ axis of oscilloscope that $* J$

$I^{*}$ represents one inch in the tank *J

float level [6500]; $I^{*}$ sample data (inches) from test*J

float rdtm[6500]; $/ *$ time at which sample was taken */

float prelevel [MAX_PRE_SAMPI; $/ *$ sample data (inches) from pre-test */

float pretm[MAX_PRE_SAMP]; $\quad I^{*}$ time at which pre-test sample was taken *I

int data_saved; . . $/ *$ true if data has been saved *J

char flname [32];

char test_flname[32]; $/$ * name of test data file *I

char opts[256]; $\quad f^{*}$ string used for menu options */

FILE *outfl;

FILE *infl;

main()

c

int quit;

int resp;

int line_number:

$f^{*}$ menu response * $/$

int line_width;

char *ptr;

*test_flname $=\prime 10^{\prime} ; \quad l^{*}$ initialize test file name to blank *

$l^{*}$ read variables from file */

sprintf(flname,"tdrvar.dat");

printf("InIn Reading variables from file \%sin", flname );

if $($ (infl $=$ fopen $($ flname, "r") ) $==$ NULL ) 
$\tau$

printf(" InIn ***k* could not open TORVAR.DAT file!!! InIn");

$\operatorname{getch}()$;

exit( 0 );

3

else

$c$

ptr $=$ fgets( strg, 32, infl );

time_period = atof( strg);

ptr = fgets( strg, 32, inf( );

base_seconds = atof( strg );

ptr $=$ fgets ( strg, 32, infl ):

base_inches $=$ atof ( strg );

ptr = fgets( strg, 32, infl);

- volt-lev-matoff istrg-r

ptr = fgets( strg, 32, infl );

ns_per_inch = atof( strg );

$3 / *$ end if file opened successfully *f

fclose (infl):

do

c

tdr_test(); $\quad J^{*}$ sample data *I

do

r

$f^{*}$ print main menu *J

_clearscreen( _GCLEARSCREEH);

resp $=1$;

sprintf(opts,

"Run; Change BASE LEVEL;G̣raph last data;Save data to floppy;Exit");

line_number $=23$;

line_width $=80$;

resp $=$ line_menu ( line_number, line_width, opts, resp );

quit $=0$;

switch ( resp)

反

case 1:

break;

case 2:

set_base_seconds(); $j *$ change base level *J

break;

case 3:

/* display graph */

system("tdrgph");

break;

case 4:

I* copy last data to a floppy *I

copy_data();

break;

case 5:

quit $=-1$;

break;

$3 / *$ end switch */

3 while ( iquit $\& \&$ resp != 1 );

3 while ( lquit): 
tdr.c

_clearscreen( _GCLEARSCREEH );

J
PAGE 3

07/01/96. 14:16:04 


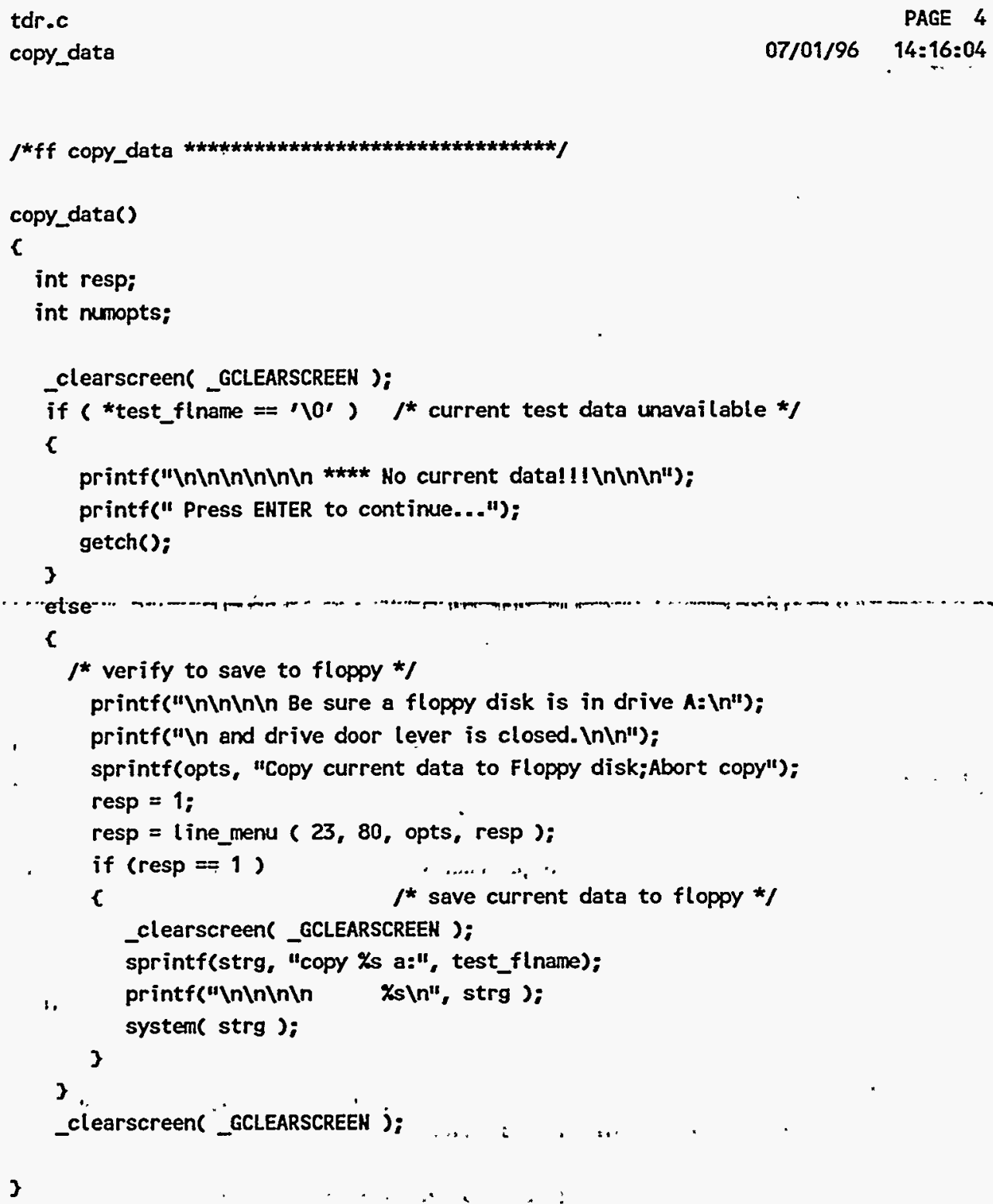


send_488( hp54121t, \&status, cmd);

sprintf(cmd,":TIMEBASE:GRATE 500000");

send_488( hp54121t, \&status, cmd);

sprintf(cmd,":DISPLAY:VMARKER OH");

send_488( hp54121t, \&status, cmd);

sprintf(cnd,":DISPLAY:TMARKER OH");

send_488( hp54121t, \&status, cmd);

sprintf( cmd, ":ACQUIRE:TYPE AVERAGE;COUNT 1");

send_488( hp54121t, \&status, cmd);

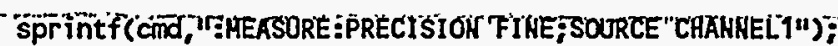

send_488( hp54121t, \&status, cmd);

ct $=0$;

prect $=0$;

$\operatorname{pretm}[$ prect] $=0$;

prelevel [prect] $=0$;

full $=0 ; \quad . \quad I^{*}$ pretest array not full *J

data_saved $=0 ; \quad f^{*}$ indicate data not saved *J

setcursor ( CURSOR_OFF ); $f^{*}$ do not display cursor *f

* determine time at voltage level */

sprintf( cmd, ":MEASURE:TVOLT? $\% f,-1 "$, volt_lev );

send( hp54121t, cmd, \&status );

_dos_getdate( \&date );

_dos_gettime( \&beg_time );

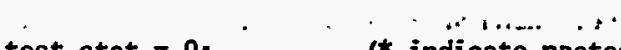

test_stat $=0 ; . \quad \quad{ }^{*}$ indicate pretest sampling $* /$

set_pre_screen();

length = 120;

enter( strg, length, \&len, hp54121t, \&status );

$/ *$ pretest sampling *

while ( test_stat $=0$ )

c

$I^{*}$ send command requesting data */

send( hp54121t, cmd, \&status );

if (status $l=0$ ) test_stat $=$ status $*-2$; .

$I^{*}$ check for trigger pulse */

pulse $=$ inp(pulse_port);

if ( pulse $I=$ pre_pulse)

c

test_stat $=1$;

/* indicate trigger received */

_settextposition( 23, 2 );

printf("

");

_settextposition( 8,10 );

printf("SAMPLING.... 
tdr.c

tdr_test
PAGE 7

07/01/96 14:16:04

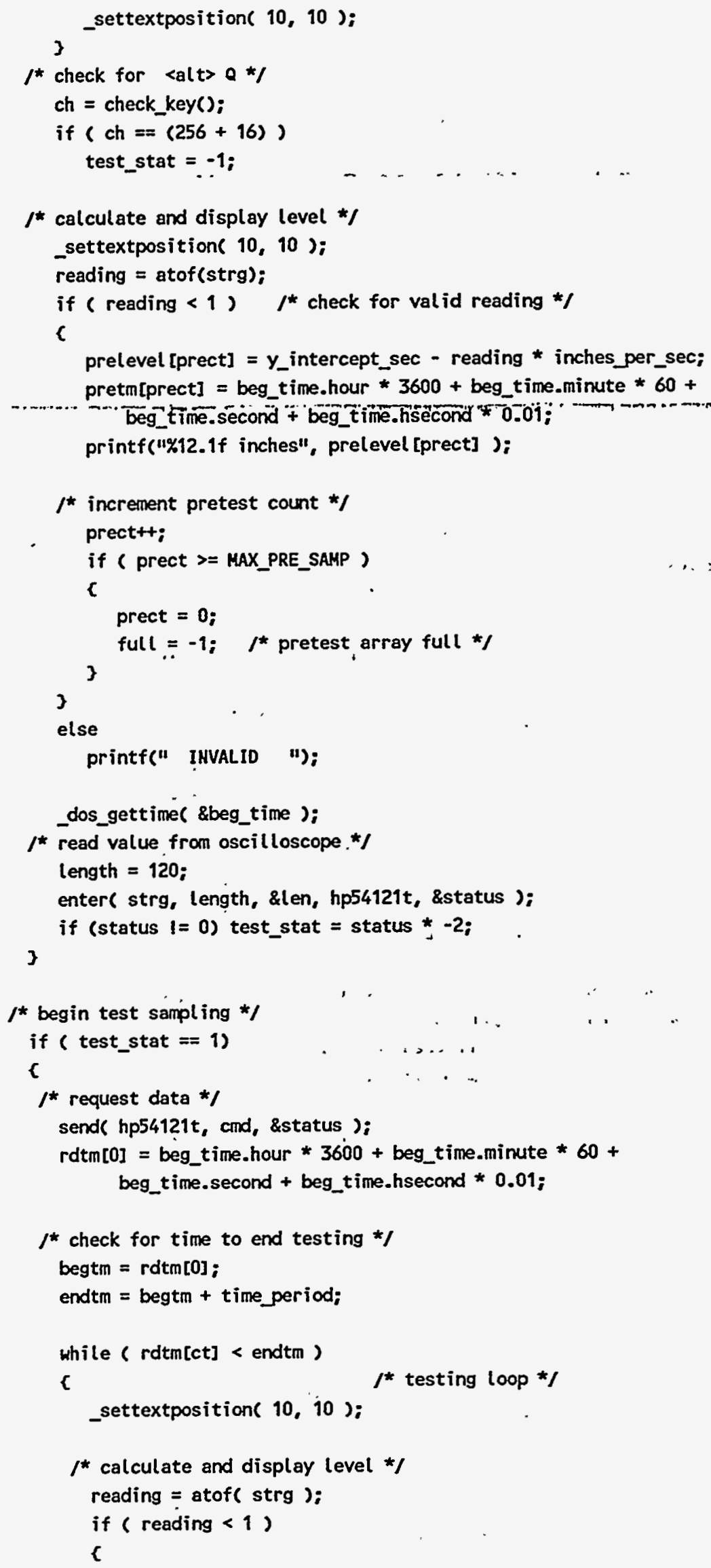




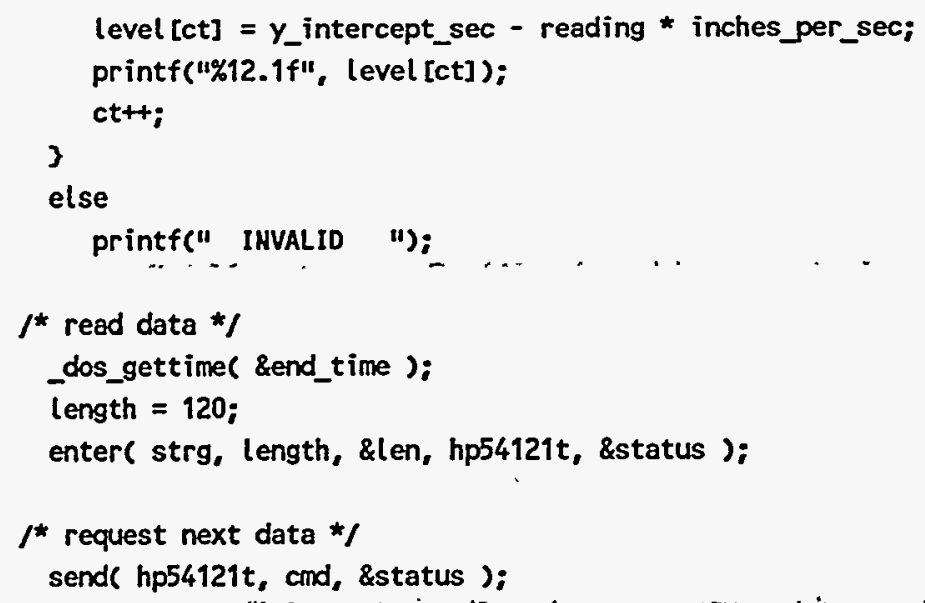


if $($ test_stat $==1) \quad /$ if save data */

c

$f^{*}$ convert data and time into data file name */

get_flname( date, beg_time, test_finame);

printf("Inln Saving data to file $\% s \backslash n "$, test_flname );

if $($ (outfl $=$ fopen ( test_flname, "w")) $=$ NULL )

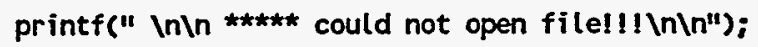

else

c

$I^{*}$ create and print title for graph */

create-tittef date; beg-time;-strg t;

fprintf( outfl, "\%sin", strg);

if ( full)

c

$i=$ prect;

if ( $i>=$ MAX_PRE_SAMP ) $i=0$;

3 num = MAX_PRE_SAMP:

else

c

$i=0$;

num $=$ prect;

3

prect $=0$;

while ( prect < num )

c

,fprintf( outfl, "\%8.3f .28.1f \n", pretm[i] - rdtm[0], prelevel[i]);

prect+t;

i++;

if $(i)=$ MAX_PRE_SAMP ) $i=0 ;$

3

for $(i=0 ; i<=c t ; i++)$

fprintf( outfl, "\%8.3f $\% 8.1$ if In", rdtm[i] - rdtm[0], (evel[i]);

fclose ( outfi ):

outfl = fopen( "datfinm.dat", "W");

fprintf(cutfl, "\%s", test_flname);

fclose( outfl);

data_saved = -1;

system( "tdrgph");

$3 / *$ end if file opened successfully */

$3 / *$ end if test run *I

sprintf(cmd, "UNL LISTEN \%d GTL", hp54121t);

transmit_488( \&status, cmd);

setcursor ( CURSOR_ON ):

$3 / *$ end tdr_test */ 
tdr.c

tdr_test
PAGE 10

07/01/96 14:16:04
. 
tdr.c

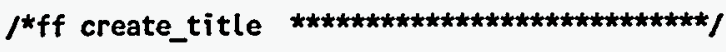

create_title( date, time, title)

struct dosdate_t date;

struct dostime_t time;

char *title;

c

char dt[30]: $/ *$ string to build date into */

char *ptr;

int $i$;

ptr $=d t ;$

sprintf( dt, "\%2d-\%2d-\%/d",

$f^{*}$ convert any spaces in date to 0 * $/$

while (*ptr $I=' 10$ ')

$c$

if $\left({ }^{*}\right.$ ptr $==1, *^{* p t r}=10^{\prime}$;

ptr++;

$j^{*}$ add spaces between date and time *j

for $(i=0 ; i<3$; $i++)$

*ptrt+ = ' ';

*ptr $=$ " $10^{\prime}$;

sprintf( ptr, "\%2d:\%2d:\%2d",

time.hour, time.minute, time.second);

$I^{*}$ convert any spaces in time to $0 * j$

while ( *ptr $1='(10 \prime)$

c

if $\left({ }^{*}\right.$ ptr $==1,{ }^{*}$ ptr $=10^{\prime} ;$

ptrt+;

3

sprintf( title, "SSS RESPONSE TEST INK LEVEL - \%s", dt);

3 
tdr.c

check_key
PAGE 12

07/01/96 14:16:04

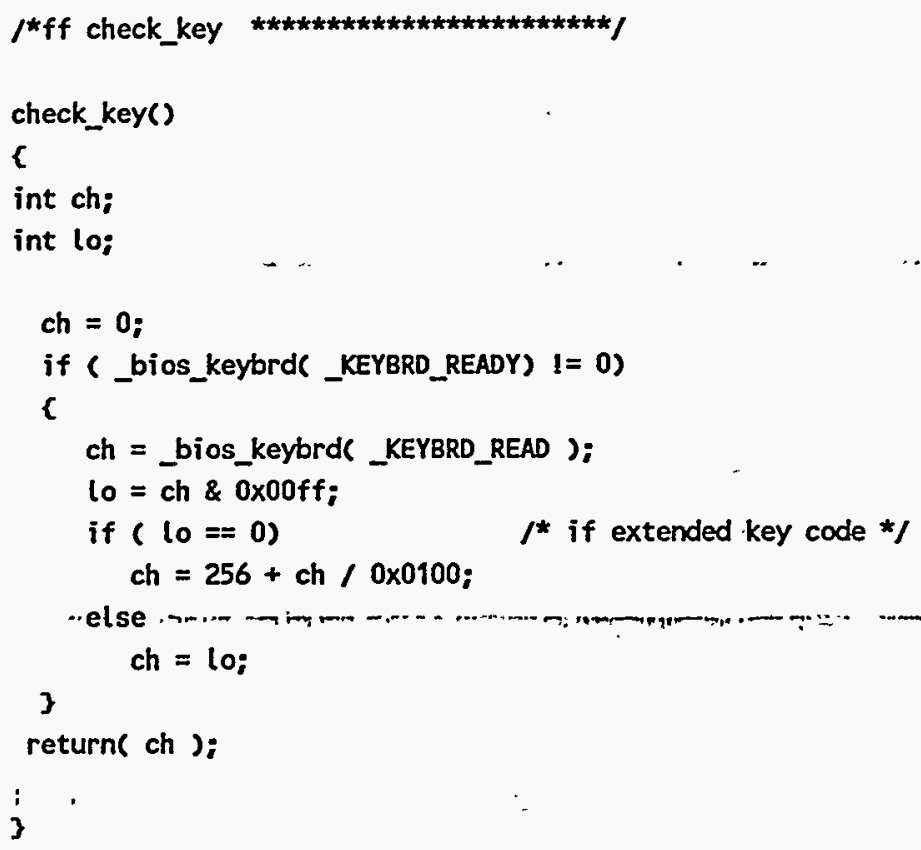




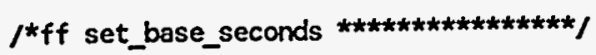

set_base_seconds( )

c

float lev; $\quad /$ * new level read *I

int status;

int length;

int len;

char cond[80];

int test_stat;

int resp;

_clearscreen( _GCLEARSCREEH );

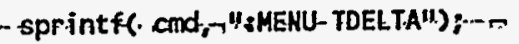

send_488( hp54121t, \&status, cmd);

sprintf( cmd, ":MEASURE:TSTART \%g", base_seconds );

send_488( hp54121t, \&status, cmd);

test_stat $=0$

sprintf(cmd, "UNL LISTEK \%d GTL", hp54121t);

transmit_488( \&status, cmd);

_clearscreen( _GCLEARSCREEN );

_settextposition( 7, 10 );

printf" base level = \%g", base_seconds );

printf("InInInin Hove Scope Delta T START MARKER ");

printf("to position $=$ \%7.1f inches. $\ln \backslash n$ ", base_inches );

resp $=1$;

sprintfopts,

"START MARKER at new base level;Exit");

resp $=$ line_menu $(23,80$, opts, resp ):

if $($ resp $=1)$

c

$l^{*}$ determine and read time indicated by operator * $/$

sprintf( cmd, ":MEASURE:TSTART?" );

send_488( hp54121t, \&status, cmd);

length $=120$;

enter( strg, length, \&len, hp54121t, \&status );

lev = atof ( strg );

if ( lev > 1) lev = base_seconds;

resp = 1;

sprintfcopts,

"Set base level = \%g seconds;Ạbort", lev);

resp $=$ line_menu $(23,80$, opts, resp );

if $($ resp $==1)$

c

test_stat $=1$; 
tdr.c

set_base_seconds

base_seconds $=$ lev;

3

3

if ( test_stat $==1) \quad / *$ new level set */

$c$

_clearscreen( _GCLEARSCREEN);

sprintf(flname,"tdrvar.dat");

if $($ coutfl $=$ fopen $($ flname, "w")) $=$ NULL $)$ printf("Inin ***** could not open file!!! In $\mid n ")$;

else

c

fprintf(outfl, "\%g\n", time_period );

fprintf(outfl, "Kgin", base_seconds );

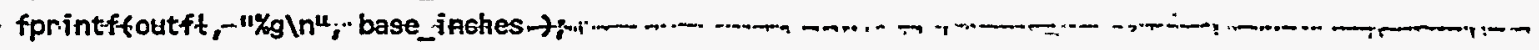
fprintf(outfl, "\%gln", volt_lev);

fprintf(outfl, "\%gln", ns_per_inch );

$3 /$ end if file opened successfully $* f$

fclose ( outfl );

$3 / *$ end if save new level *f

_clearscreen( _GCLEARSCREEN );

$3 / *$ end set_base_seconds *I
PAGE 14

07/01/96 14:16:04 
/*ff set_pre_screen

set_pre_screen()

c

_clearscreen( _GCLEARSCREEN );

printf(" Test will run for $\% 5.0$ f seconds after trigger. In (n", time_period); printf(" voltage lèvel $=\chi_{g}$ voltssini, volt _lev);

printf(" ns per inch = Kg (n", ns_per_inch);

printf(" base level: $\% 7.1 \mathrm{f}$ inches at $\bar{\chi}_{g}$ seconds $I n "$,

base_inches, base_seconds);

settextposition( 23, 2 );

printf("<ALT> a -Menu");

- settextposit-ion $(-8,-10 \cdots)$

printf("PRETEST SAMPLING....");

3 


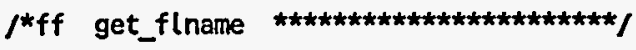

get_flname( date, time, flname )

struct dosdate_t date;

struct dostime_t time;

char *flname;

$c$

char *ptr;

ptr = flname;

${ }^{\text {*ptrt+ }}=101+$ (int)(date.month $/ 10$ );

*ptrt+ = $10 \prime$ + date.month \% 10;

*ptrt+ $=10 \prime+$ (int) (date.day $/ 10)$;

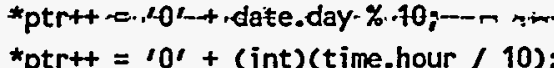

*ptrt+ $=10 \prime+$ time.hour $\% 10$;

*ptr+t $=10 \prime+$ (int) (time.minute $/ 10)$;

${ }^{*}$ ptrt+ $=10 \prime+$ time.minute $\% 10$;

${ }^{*}$ ptr $=$ '>0';

sprintf( ptr, ".tdr"); 
tdr.c

transmit_488

$j^{*}$ ff transmit_ 488

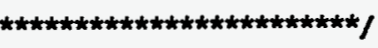

transmit_ 488 ( status, cmd )

int *status;

char *and;

c

transmit( cmd, status );

if $(*$ status $t=0)$

c

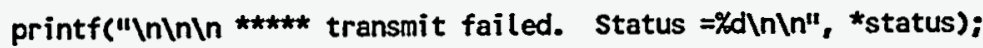

printf(" conmand = >\%s< $>n \backslash n \backslash n "$ " cmd );

exit(1);

3

-............. 
tdr.c

send_488
PAGE 18

07/01/96 14:16:04

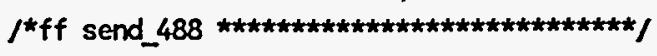

send_488( addr, status, data )

int addr:

int *status;

char *data;

c

send( addr, data, status );

if ( *status $!=0$ )

C

printf(")n Inln ***** send failed.");

printf("InIn Address =\%d, status =\%d InIn", addr, *status);

... -..printf(" -command- $\Rightarrow \%$ s $<$ tnintn", -data $\lambda_{1}$ exit(1);

3

3 
FIIE: menu.c

Iine_menu

extkey

setcursor

$10 \mathrm{C}$

color_string

disp_box
2

5

6

7

8

9 
\#include 〈stdio.h> \#include <ctype.h> \#include <time.h> \#include <conio.h> \#include <stdlib.h> \#include <limits.h> \#include <float.h> \#include <bios.h> \#include 〈dos.h>

\#include emath.h>

\#include "menu.h" $/ *$ defines for menu constants */

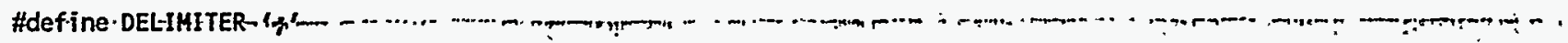
\#define HINDOW_HIDTH 40

char strg[82]; 


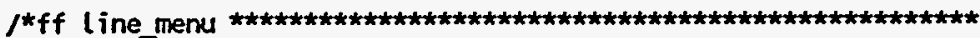

* FUnCTIOH: line_menu

* Menu recieves a string for the options.

* The string is divided at the DELIMITER to form

* the different options.

* The options are separated by DELIMITERs and should begin with a

* uppercase letter. Do not put blanks after the DELIMITER. The

* options sill all be on the same line.

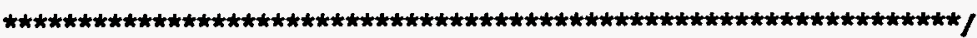

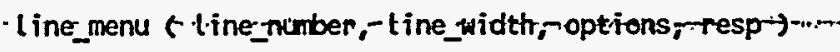

int line_number; $I^{*}$ display mode */

int line_width;

char *options; $/ *$ menu options *f

int resp; $l^{*}$ default response and response returned *J

c

char *ptr;

char *opts [20]; $I^{*}$ options */

int numopts; $\quad f^{*}$ number of options */

register int $i$;

int cur_col; " $J^{*}$ current colum */

int optcur [20]; $J^{*}$ options column location *J

int quit;

1.. i

int selected; $\quad \boldsymbol{J}^{*}$,indicates an option selected */

, int ch;

int extcode; . !* extended keyboard code */. . , . . :

int prevresp; $j *$ previous response $* j \quad \ldots \ldots \ldots \ldots \ldots$

int length;

char chopt [201;

$f^{*}$ first character of option *J

int chet;

loc_cursor ( lịne_number, 1 ); ‥ ‥

cur_col = 1:

disp_box ( line_number, cur_col, 1, line_width - 1 , BRIGHT_INTENSITY, HHITE, BLUE);

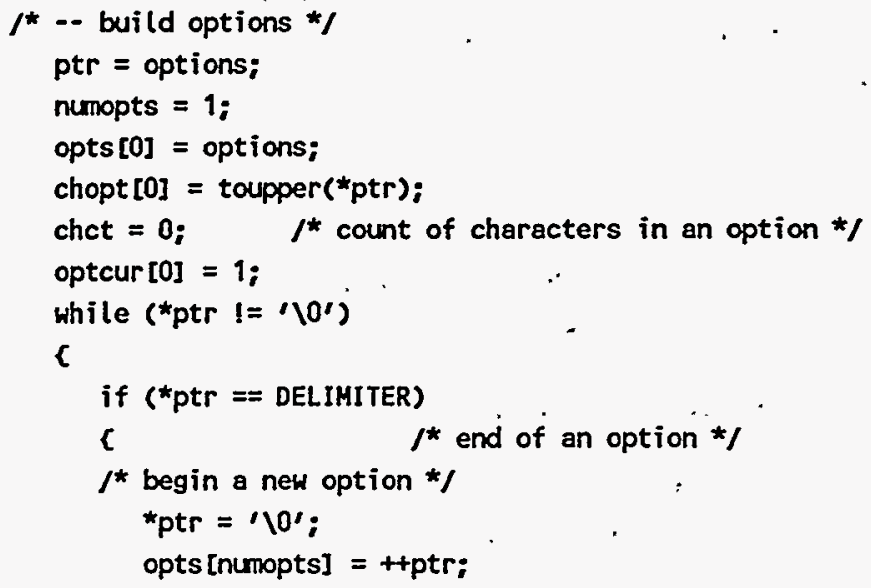




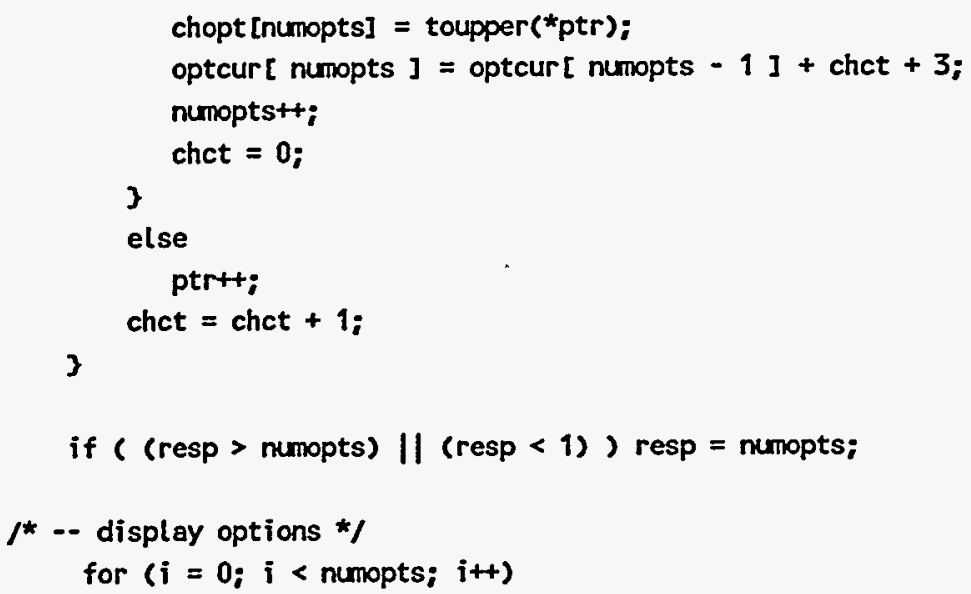


if $(c h>0) \quad / *$ if normal key pressed */

if (isalnum(ch))

反 ch = toupper(ch);

$/$ * compare key value to first character of options */ $i=$ prevresp; if ( $i$ >= numopts) $\hat{i}=0$; $^{-}$ while (ch $I=$ chopt [i] \&\& $i l=($ prevresp - 1$)$ )

c

$$
\text { it+; }
$$

if ( $i>=$ numopts) $i=0$;

3

if $($ ch $==$ chopt $[i])$ resp $=i+1$;

了

-.... If (resp-! $!$-prevresp)

c

/* re-display last option */

loc_cursor (line_number, optcur [prevresp - 1]);

color_string(NORMAL_INTENSITY,RED,BLUE, opts [prevresp - 1])

$/$ * display nek option */ loc_cursor (line_number, optcur [resp - 1]);

3 color_string ( BRIGHT_INTENSITY, UHITE, BLUE, opts[resp - 1$]$ );

- $3 /$ end while EHTER not preșsed */ cur_col $=1$; disp_box ( line_number, cur_col, 1 , îne_width - 1 , NORMAL_INTENSITY, RED, BLUE);

setcursor ( CURSOR_OH ); return (resp);

3 


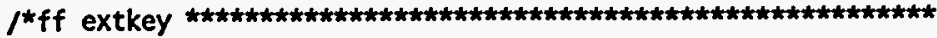

*

* Extended key checks for a keystroke at the keyboard. If a key

* has been pressed the character is returned. If a key using

* the extended codes is pressed, 0 is returned and the extended

* code is returned in extcode. If no key has been pressed, -1

* is returned.

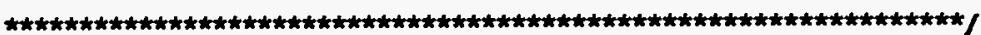

extkey ( extcode)

int *extcode; $\quad I^{*}$ extended key code */

c

-.unsigned int-chint:

int loch; $\quad$ * returned value */

Loch = -1;

*extcode $=0$;

if (_bios_keybrd(_KEYBRD_READY) !=0 )

c

/ key has been pressed */

chint $=$ bios_keybrd(_KEYBRD_READ):

loch $=$ (chint \& Oxff)

*extcode $=($ int $)($ chint $/$ oxff $)$;

3

return (loch);

3

\#define VIDEO $0 \times 10$

\#define CURSIzE 1

\#define STOPBIT 0x20

inion 'REGS inregs;

union REGS outregs;

\#define CURSIZE 1. \#define sTOPBIT 0x20 


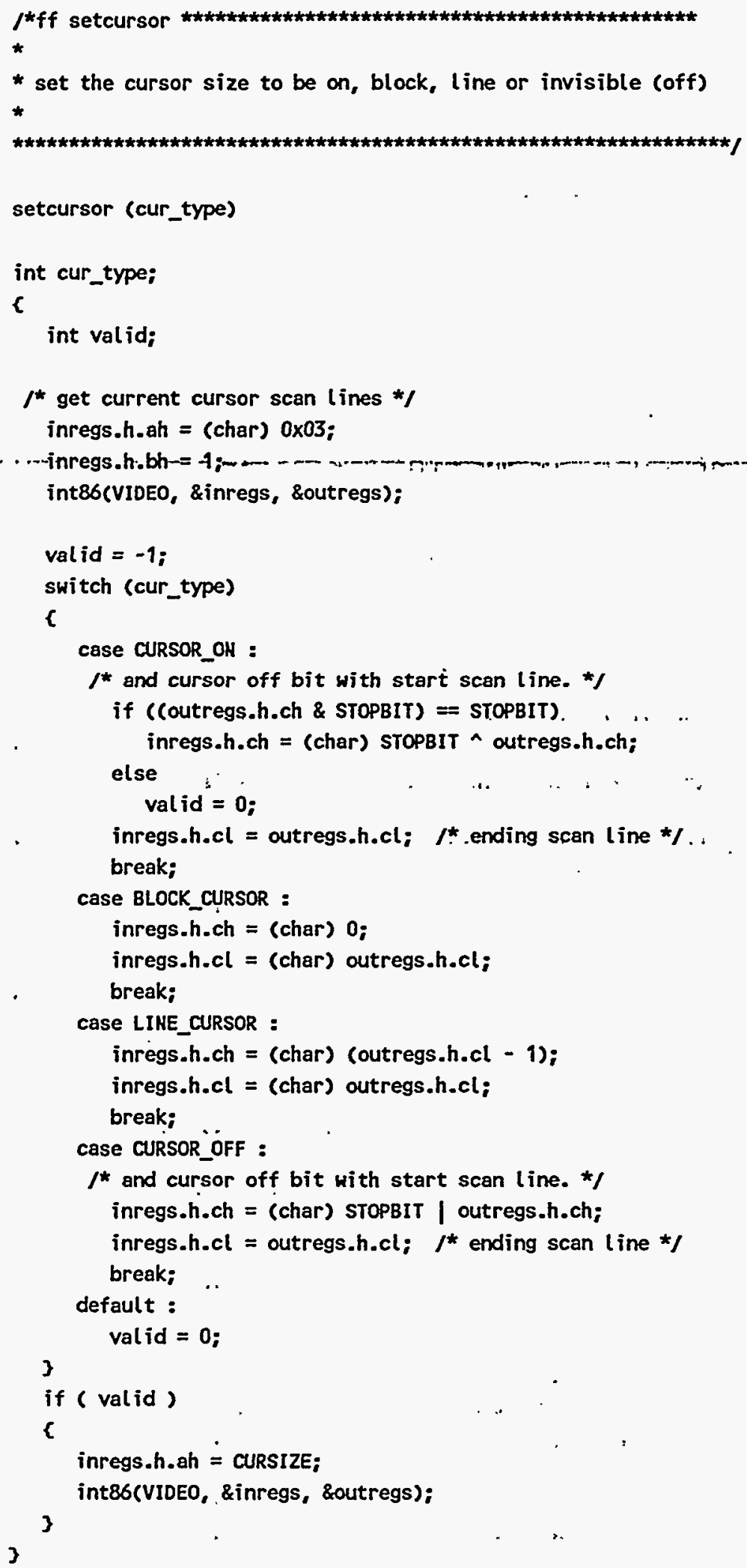




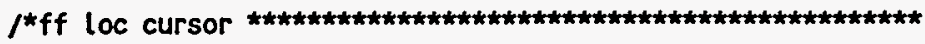

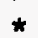

- Locate cursor on screen

$\star$

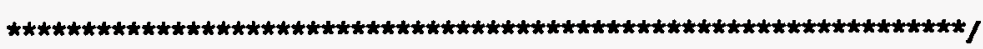

loc_cursor( row, column)

int row;

int colum;

$c$

inregs.h.bh $=$ (char) 0 ;

inregs.h.ah $=2$;

-. - inregs.h.dh $=$ row; -

inregs.h.dl = colum;

int\&6(VIDEO, \&inregs, \&outregs );

3 


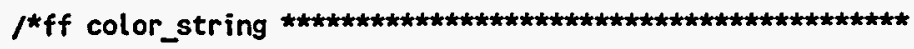

*

* display string in the intensity, foreground color and

* background color specified.

$\star$

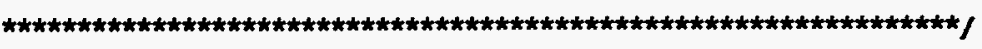

color_string ( intensity, foreground, background, strg )

int intensity;

int foreground;

int background;

char *strg;

c

"char-ch; -

int attribute_val;

intensity $=$ (intensity \& 1) $\ll 3$;

attribute_val = intensity $\mid$ foreground $\mid$ (background $\ll 4$ );

ch $=$ ' ';

while (*strg $\left.!=\wedge 10^{\prime}\right)$

c

/* change the character attribute *f . ... . ...

inregs.h.bh $=$ (char) 0 ;

inregs.h.ah = (char) 9;

inregs.h.al $=$ ( char $) '$ ';

- inregs.h.bl $=$ ( char $)$ attribute_val;

inregs.x.cx = 1;

/* print 1 çharacter */.

int86(VIDEO, \&inregs, \&outregs );

if ( $\left(\right.$ ch $I=\prime\left(r^{\prime}\right)$ \&\& (*strg $==\left(\backslash n^{\prime}\right)$ )

ch $=$ ' $r^{\prime} ; \quad \rho^{*}$ send carriage return in addition to line feed *l else

r

ch = *strg;

strg++;

3

inregs.h.bh = 0;

inregs.h.ah $=0 \times 0 E$;

inregs.h.al $=($ char $) \mathrm{ch}$;

int86(VIDEO, \&inregs, \&outregs );

3

3 


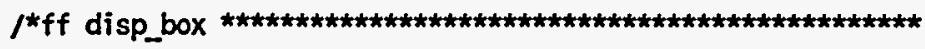

*

* displays colored box on screen

*

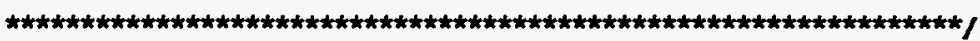

disp_box ( start_line, start_colum, num_linès; line_length, intensity, foreground, background)

int start_line; $\quad / *$ line of screen box starts on *J

int start_colum; $f^{*}$ column of screen box starts on */

int num_lines; $\quad / *$ number of lines. in box */

int line_length; $f^{*}$ length of each line *f

int intensity; $\quad / *$ color intensity - $0=$ normal $1=$ bright *I

int foreground; $-m f^{*}$ rcolor- of characters $\rightarrow=0$-thru- $7 . * y$

int background; $\quad I^{*}$ color of background - 0 thru 7 *J

$c$

int attribute_val; $/$ * value of attribute calculated *J

register int $\bar{i}$;

/* convert intensity to bit $3 * /$

intensity $=$ (intensity \& 1$) \ll 3$;

$I^{*}$ determine attribute value from intensity, foreground color and background color */

$\because \quad$ attribute_val = intensity $\mid$ foreground $\mid$ (background $\ll 4$ );

for ( $i=0$; $i<$ num_lines; it+)

c

loc_cursor(start_line $+i$, start_colum);

inregs.h.bh $=$ (char) 0 ;

inregs.h.ah $=$ (char) 9;

inregs.h.al $=$ ( char $)$ ' ';

inregs.h.bl = ( char ) attribute_val;

inregs.x.cx = line_length;

int86(VIDEO, \&inregs, \&outregs ); . . ...

$i^{3}$

3 
\#define BLACK 0

\#define BLUE 1

\#define GREEN 2

\#define CYAN 3

\#define RED 4

\#define MAGENTA 5

\#define BROWH 6

\#define WHITE 7

$f^{*}$ use bright intensity to get yellow instead of brown */

\#define YELLOW 6

$J$ * intensity values *

\#define NORHAL_INTENSITY 0

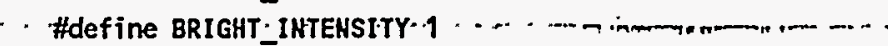

\#define HIDTH_40_MODE 1

\#define HIDTH_80_MOOE 3

\#define SCREEN_HIDTH 80;

\#define UP_ARROW

\#define DHN_ARROW

$72 I^{*}$ ascii codè for up arron key *J

\#define LFT_ARROW

$80 J^{*}$ ascii code for down arrow key */

\#define RT_ARROW

$75 / *$ ascii code for left arrow key *J

$77 / *$ ascii code for right arrow key *J

1 * defines for display mode of menu program */

\#define HORMAL_MENU 0

\#define TIME_MENU $2 / *$ Displays time and date on top line */

$1 *$ defines for cursor type */

\#define CURSOR_ON 0

\#define LIME_CURSOR 1

\#define BLOCK_CURSOR 2

\#define CURSOR_OFF: . 4 